\title{
Congenital rubella: Is it nearly time to take action?
}

\author{
L Boshoff, L Tooke \\ Department of Paediatrics: Neonatology, Groote Schuur Hospital and University of Cape Town \\ L Boshoff, MB ChB, DCH (SA) \\ L Tooke, MB ChB, FCPaeds (SA), MMed (Paed), Cert Neonatol, Dip PEC, Dip Obst
}

Corresponding author: L Boshoff (lizeboshoff@hotmail.com)

Congenital rubella syndrome (CRS) is a rare but potentially debilitating disease with lifelong consequences. Although there is no cure, it is almost completely preventable by an effective immunisation programme. We present two confirmed cases of CRS diagnosed in the neonatal unit at Groote Schuur Hospital in 2011 and discuss aspects of the disease and its prevention.

S Afr J CH 2012;6(4):106-108. DOI:10.7196/SAJCH.461

The exact incidence of congenital rubella syndrome (CRS) is not known in South Africa, but it is estimated that approximately 660 cases occur annually. Acquired and congenital rubella are not notifiable diseases in this country, and epidemics often go unnoticed. Although classically a triad of cataracts, deafness and cardiac disease, the clinical manifestations of CRS vary from mild and unrecognised to severely debilitating. Congenital rubella is completely preventable by having an effective immunisation programme in place. Currently routine rubella vaccination does not form part of the Expanded Programme for Immunization (EPI) in South Africa. The availability of the vaccine in the private sector may be setting our population up for a surge in new cases of CRS.

In 2011 there were 2 confirmed cases of congenital rubella in the Neonatal Unit at Groote Schuur Hospital, Cape Town.

\section{Case reports \\ Case 1}

A baby weighing $1980 \mathrm{~g}$ and scored at 36 weeks' gestation using the modified Ballard score ${ }^{2}$ was born to a 14-year-old mother who despite her young age had been pregnant previously and was well known to the social services. She had booked late but was HIVand RPR-negative. She reported no viral illness or rash early in the pregnancy.

Because of the unexplained preterm labour and mild respiratory distress the baby was admitted to the neonatal nursery, a septic screen was done and first-line antibiotics were started. Of further concern on clinical examination was symmetrical growth restriction, the presence of leukocoria of the left eye and a systolic murmur. There was no associated rash or microcephaly. A blood sample was sent to screen for congenital infections including rubella.

On day 3 of life the baby was noted to be lethargic with a slightly full fontanelle. An ultrasound scan of the head showed no hydrocephalus or structural abnormalities. Septic markers were negative, but the antibiotics were continued. On day 4 the baby had an acute decompensation, requiring resuscitation, intubation and ventilation. Blood gas measurements showed marked metabolic acidosis with a raised lactate level. Multi-organ involvement manifested as renal impairment, raised liver transaminases and disseminated intravascular coagulation with thrombocytopenia. Repeat septic markers and culture remained negative. Formal echocardiography ruled out coarctation of the aorta, but diagnosed a large patent ductus arteriosus (PDA) with no evidence of cardiac failure. Continuous cerebral function monitoring (CFM) showed burst suppression on the trace. Despite full supportive care and review of the antibiotic cover, there was no neurological improvement after 96 hours and the CFM readings continued to show a background trace consistent with poor outcome. After consultation with the mother and grandmother, the decision was made to de-escalate therapy and the baby died 12 hours after extubation. Serum rubella IgM and urine rubella polymerase chain reaction (PCR) confirmed the diagnosis of rubella, and cerebrospinal fluid PCR (sent postmortem) demonstrated that rubella meningo-encephalitis was present.

\section{Case 2}

A baby weighing $2190 \mathrm{~g}$ was delivered at term via caesarean section for fetal distress. His mother was a 20-year-old primigravida who reported no rash or viral illness during her pregnancy but did have contact in the early months with a child who had a rash suggestive of a viral illness.

The baby was transferred to the neonatal nursery for further management. Clinical examination revealed symmetrical growth restriction, leukocoria of the left eye (later confirmed as a cataract), and a systolic murmur suggestive of a PDA.

Serum rubella IgM and urine rubella PCR were positive. A hearing screen before discharge was inconclusive, and the baby was discharged on day 4 with follow-up appointments at the cardiology, audiology, ophthalmology and neurodevelopmental clinics. The mother was referred to the social worker.

\section{Discussion}

Rubella was first recognised as a teratogen in 1941 when Gregg, an Australian ophthalmologist, associated intra-uterine infection with the development of cataracts and other defects. ${ }^{3}$ Classically CRS leads to the triad of deafness (80\%), congenital cardiac disease (50 - 70\%, most commonly PDA or pulmonary stenosis) and cataracts (30\%), but many other defects involving almost every organ have been described ${ }^{4}$ (Fig. 1). 


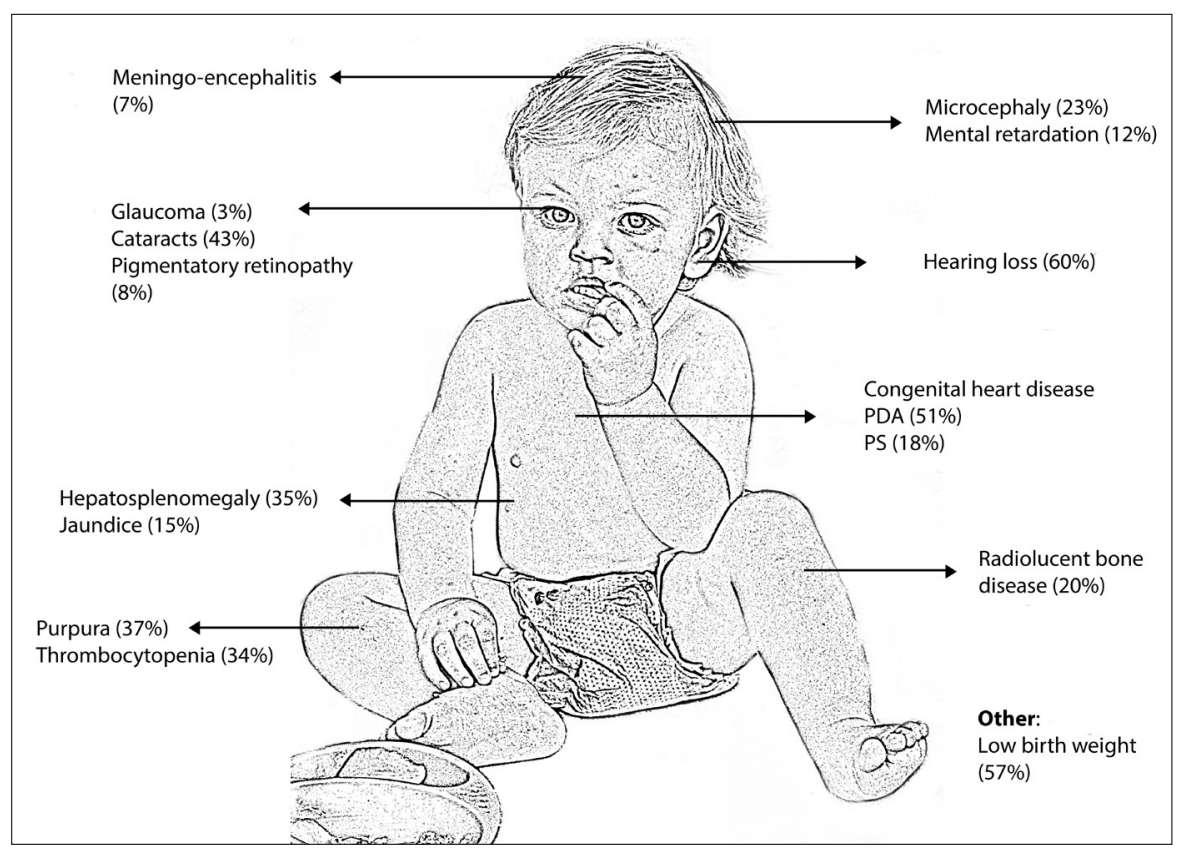

Fig. 1. Common clinical manifestations of congenital rubella syndrome.

The risk is highest if the mother is infected in the first trimester of her pregnancy. Infection during this time carries a risk of CRS that can be as high as $90 \%$, although spontaneous miscarriage is common..$^{5-7}$ Sensorineural deafness can occur with infection right up to the 19th week of gestation and may only become evident later in childhood. ${ }^{8}$ More severe complications include acute meningo-encephalitis (10 - 20\%) and lateonset progressive panencephalitis. The risk of intellectual disability, behavioural problems and autism are all increased in children with CRS. Studies have shown that affected adults also have an increased risk of developing endocrinopathies such as diabetes mellitus and thyroid problems. ${ }^{4}$ Although CRS is a disease with a variable spectrum of clinical presentation and outcome, many patients require lifelong care. In the USA the lifetime cost of treating a patient with CRS is estimated to be more than $\$ 200000 .^{9}$

Congenital rubella is not a notifiable disease in South Africa, but it is estimated that about 660 cases of CRS occur in this country every year. The global incidence is in the region of 100000 cases annually, mostly in developing countries. ${ }^{1,10,11}$ Epidemics usually occur every $4-7$ years, but as there are no surveillance programmes in place, these outbreaks can occur without clinical recognition. ${ }^{12}$

Although screening for rubella antibodies in pregnancy is widespread in the private healthcare sector, no routine antenatal seroprevalence screening takes place in the public sector. However, a study by Corcoran and Hardie in the Western Cape found that $95.3 \%$ of women in the 15 - 24-year age group, $97.5 \%$ in the $25-34$-year age group and $98 \%$ in the 35 - 45-year age group were immune to rubella. ${ }^{13}$ Interestingly, Schoub et al. demonstrated a susceptibility gap (rubella IgG-negative) for women 12 - 49 years of age in the private sector that was significantly greater than that in the public sector $(10.7 \%$ v. $5.4 \%)$. This can probably be attributed to better socio-economic circumstances, less overcrowding, and partial immunisation of the population.

In the affected infant, rubella can be confirmed by isolating virus from a nasopharyngeal swab, or PCR can be done on body fluids - urine is the easiest to collect. Infected infants can shed virus for up to 6 12 months after birth, and occasionally for longer. Rubella-specific IgM can usually be detected in the first 6 months, and with the clinical picture of CRS is highly suggestive of prenatal infection. Rubella-specific IgG beyond 6 months can be detected in $95 \%$ of infants with CRS but may also be caused by postnatal infection or passively transferred maternal antibodies. This is what makes retrospective diagnosis of CRS in an older infant or child more difficult. ${ }^{8,14,15}$

Currently no definitive treatment for CRS exists and there is no role for passive immunisation with immune globulin after maternal exposure.Termination of pregnancy in confirmed cases of first-trimester infection should be discussed with parents after appropriate counselling, preferably by a specialist in congenital infectious diseases. Once a baby with CRS is born, isolation precautions should be instituted and these children should be considered infectious for the first year of life. Susceptible female healthcare workers or visitors should be informed of the potential risk of exposure. The use of chemotherapeutic agents such as amantadine, interferon and isoprinosine has shown no benefit. ${ }^{4}$

CRS is a chronic disease, and these children need to be followed up to detect progression and the emergence of new problems. A multidisciplinary team approach is required, involving medical, surgical, educational and rehabilitative management.

\section{Vaccination}

As rubella is typically a mild disease, the primary purpose of rubella vaccination is to prevent congenital rubella infection. The current vaccine is a live attenuated vaccine and contains the RA27/3 strain. It is safe, cheap and effective and can be administered either as a monovalent vaccine, or in combination with the measles (MR) or measles and mumps (MMR) vaccines. ${ }^{11}$ Vaccine-induced protection is generally assumed to be lifelong and studies indicate that over $90 \%$ of vaccinees are protected for at least 15 years. ${ }^{16}$

Rubella vaccine is highly affordable, with the cost between $\$ 0.31$ and $\$ 1.37$ depending on the formulation. ${ }^{17}$ Owing to the high cost of treating infected individuals, cost-benefit analyses of vaccination in both developed and developing countries have shown ratios $>1 .{ }^{9}$

However, rubella vaccination does not currently form part of the EPI in South Africa, although parents can have their children immunised in the private sector as part of the MMR vaccine, which can be given at 15 months, with a booster at age 5 years.

In 2000 the World Health Organization (WHO) published its first rubella vaccination position paper to guide the introduction of rubella-containing vaccine $(\mathrm{RCV})$ in national childhood immunisation schedules. From 1996 to 2009, the number of countries that introduced $\mathrm{RCV}$ into their national routine immunisation programmes increased by $57 \%$. Many developing countries in South America, North Africa and parts of Asia now have rubella vaccination as part of their schedules. ${ }^{17}$ However, RCV is not recommended for inclusion in the EPI of developing countries when sustained high immunisation coverage cannot be maintained, as this could increase the susceptibility of adult women by slowing, but not interrupting, rubella transmission and thereby theoretically actually increase the number of congenital rubella cases..$^{16,18-20}$ The WHO recommends first-dose measlescontaining vaccine coverage (MCV1) of $>80 \%$ before RCV is introduced. ${ }^{11}$ In South Africa, all but 8 districts currently meet this criterion for the first dose of measles 
vaccine. ${ }^{21,22}$ The Department of Health is once again engaging in the WHO's 'Reaching Every District' (RED) strategy to ensure coverage across the whole country. ${ }^{23}$

The use of MMR in the private sector may be contributing to a population of susceptible young women. ${ }^{20,24}$ It is estimated that about 100000 children per year utilise private vaccination facilities, with $60 \%$ of these receiving rubella vaccination. This is significant enough to create an immunity gap in this population. ${ }^{1,25}$

A combined immunisation strategy has been found to be the most effective method of eradicating CRS. The immunisation of all infants will only eradicate CRS in 30 - 40 years and that of all schoolgirls presumably in 10 - 20 years. Universal childhood vaccination alone is not the recommended vaccination strategy - not only is the lag phase unacceptably long, but there is a risk of increased CRS cases initially, as seen in Greece in 1993..$^{14,20}$ Vaccination of young women will eradicate CRS immediately, but only if $100 \%$ are immunised.

As the median age of sexual debut in South Africa is 16 - 17 years, an option would be to introduce vaccination to prepubescent girls, preferably at primary school, as part of the school health services. ${ }^{26}$ At present regular healthcare visits at primary school are not well established, but this may change with the planned introduction of the combined diphtheria and tetanus vaccine (and potentially the human papillomavirus (HPV) vaccine) at age $12 .{ }^{27}$ High rates of female school attendance and good liaison between school and health authorities will be required.

\section{Conclusion}

In South Africa the exact burden of CRS is unknown. As CRS is potentially a preventable disease, although rare, every new case is not only a tragedy for the family but a failure of the health system.

Rubella vaccine is safe, effective and cheap with a favourable costbenefit ratio. There is potential to introduce the vaccine into the EPI. With careful planning it could be introduced at the same time as the HPV vaccine.

Although South Africa has many more pressing health and social issues, a marked reduction or even elimination of CRS cases is possible. It is to be hoped that in the future we will see even fewer cases of CRS in our units.

We are interested in finding out how many others have diagnosed cases of congenital rubella, and invite readers to send information to the corresponding author.

\section{References}

1. Schoub BD, Harris BN, McAnerney J, Blumberg L. Rubella in South Africa: An impending Greek tragedy? S Afr Med J 2009;99(7):515-519.

2. Ballard JL, Khoury JC, Wedig K, et al. New Ballard score expanded to include extremely premature infants. J Pediatr 1991;119:417-423. [http:// dx.doi.org/10.1016/S0022-3476(05)82056-6]

3. Gregg NM. Congenital cataract following German measles in mother. Transactions of the Opthalmological Society of Australia 1941;3:35-36. [http://dx.doi.org/10.1017/S0950268800048627]

4. Remington JS, Klein JO, Wilson CB, Nizet V, Maldonado YA. Infectious Diseases of the Fetus and Newborn Infant. 7th ed. Philadelphia: Elsevier Saunders, 2011:861-898.

5. Best JM, O'Shea S, Tipples G, et al. Interpretation of rubella serology in pregnancy - pitfalls and problems. BMJ 2002;325:147-148. [http://dx.doi. org/10.1136/bmj.325.7356.147]
6. Miller E, Cradock-Watson JE, Pollock TM. Consequences of confirmed maternal rubella at successive stages of pregnancy. Lancet 1982;2:781-784. [http://dx.doi.org/10.1016/S0140-6736(82)92677-0]

7. Siegel M, Fuerst HT, Peress NS. Fetal mortality in maternal rubella: results of a prospective study from 1957-1964. Am J Obstet Gynecol 1966;96:247253.

8. Cooper LZ. The history and medical consequences of rubella. Reviews of Infectious Diseases 1985;7(1):S2-S10.

9. Hinman AR, Irons B, Lewis M, Kandela K. Economic analysis of rubella and rubella vaccines: a global review. Bull World Health Organ 2002;80:264-270.

10. Cutts FT, Vynnycky E. Modelling the incidence of congenital rubella syndrome in developing countries. Int J Epidemiol 1999;28:1176-1184. [http://dx.doi.org/10.1093/ije/28.6.1176]

11. World Health Organization. Rubella vaccines. WHO Position Paper. Wkly Epidemiol Rec 2011;86:301-306.

12. Cutts FT, Robertson SE, Diaz-Ortega JL, Samuel R. Control of rubella and congenital rubella syndrome (CRS) in developing countries, Part 1: Burden of disease from CRS. Bull World Health Organ 1997;75(1):55-68.

13. Corcoran C, Hardie DR. Seroprevalence of rubella antibodies among antenatal patients in the Western Cape. S Afr Med J 2005;95:688-690.

14. Plotkin SA. Rubella vaccine. In: Plotkin SA, Mortimer EA, eds. Vaccines. 2nd ed. London, WB Saunders, 1994.

15. Plotkin SA, Cochran W, Lindquist JM, Cochran GG, Schaffer DB, Harold G. Congenital rubella syndrome in late infancy. JAMA 1967;200(6):435441. [http://dx.doi.org/10.1001/jama.1967.03120190061007]

16. Robertson SE, Cutts FT, Samuel R, Diaz-Ortega JL. Control of rubella and congenital rubella syndrome (CRS) in developing countries, Part 2: Vaccination against rubella. Bull World Health Organ 1997;75(1):69-80.

17. Reef SE, Strebel P, Dabbagh A, Gacic-Dobo M, Cochi S. Progress toward control of rubella and prevention of congenital rubella syndrome worldwide, 2009. J Infect Dis 2011;204(suppl 1):S24-S27. [http://dx.doi.org/10.1093/infdis/jir155]

18. WHO Global Programme for Vaccines and Immunization. Immunization policy. WHO/EPI/GEN/95.03/Rev.1, 1996. Geneva: World Health Organization, 1996. http://www.who.int/iris/handle/10665/63114 (accessed 10 November 2012).

19. Anderson RM,May RM. Vaccination against rubella and measles: quantitative investigations of different policies. Journal of Hygiene 1983;90:259-325. [http://dx.doi.org/10.1017/S002217240002893X]

20. Panagiotopoulis T, Antoniadou I, Valassi-Adam E. Increase in congenital rubella occurrence after immunization in Greece: Retrospective survey and systematic review. BMJ 1999;319:1462-1467. [http://dx.doi.org/10.1136/ bmj.319.7223.1462]

21. World Health Organization. Report of the second meeting of the African Regional Measles Technical Advisory Group (TAG), recommendations. Addis Ababa, Ethiopia: World Health Organization, Regional Office for Africa, 2008. http://www.afro.who.int/en/search.html? searchword=Measles +TAG\&ordering=\&searchphrase=all (accessed 11 November 2012).

22. Masresha BG, Fall A, Eshetu M, et al. Measles mortality reduction and preelimination in the African region, 2001 - 2009.J Infect Dis 2011;204(suppl 1):S198. [http://dx.doi.org/10.1093/infdis/jir116]

23. World Health Organization. The RED strategy. http://www.who.int/ immunization delivery/systems policy/red/en (accessed 12 March 2012).

24. Johnson S, McAnerney JM, Schoub BD, Kidd AH. Laboratory monitoring of rubella. S Afr Med J 1985;67:721-723.

25. Vynnycky E, Gray NJ, Cutts FT. The predicted impact of private sector MMR vaccination on the burden of congenital rubella syndrome. Vaccine 2003;1(2122):2708-2719. [http://dx.doi.org/10.1016/S0264-410X(03)00229-9]

26. Richter L, Panday S, Emmet T, et al. The status of youth report 2003: young people in South Africa, 2005. http://www.hsrc.ac.za/Research_ Publication-18907.phtml (accessed 14 November 2012).

27. Harries J, Moodley J, Barone MA, Mall S, Sinanovic E. Preparing for HPV vaccination in South Africa: Key challenges and opinions. Vaccine 2009;27:38-44. [http://dx.doi.org/10.1016/j.vaccine.2008.10.033] 